\title{
O Inquérito eletrocardiográfico
}

\author{
The electrocardiographic survey
}

\author{
José Geraldo Ferreira Gonçalves*, Aluízio Prata**, João Carlos Pinto Dias*** e Vanize Macêdo†
}

\begin{abstract}
RESUMO
Entre 1977 e 1981, foi realizado um inquérito eletrocardiográfico nacional tendo por objetivo avaliar a prevalência da cardiomiopatia chagásica no Brasil. Foram feitos 5.347 eletrocardiogramas. Após o pareamento dos indivíduos, segundo a idade e gênero, a distribuição dos eletrocardiogramas dos autóctones, em cada estado, ficou assim constituída: Rio Grande do Sul (1.078), Minas Gerais (760), Bahia (612), Paraná (400), Paraíba (340), Piauí (218), Sergipe (216), Goiás (176), Pernambuco (170), Ceará (136) e Alagoas (134). Os maiores percentuais de alterações eletrocardiográficas entre os indivíduos com soros reagentes foram encontrados nos Estados de Goiás (63,6\%), Minas Gerais (57,6\%), Ceará (57,3\%), Paraná (54,5\%), Piauí (53,2\%) e Paraíba (52,3\%). Entre os controles, nestes estados, a prevalência das alterações eletrocardiográficas foi de $25 \%, 25,7 \%, 25 \%, 12,5 \%, 22,9 \%$ e 26,5\% respectivamente. Observou-se diferença estatisticamente significativa em relação à prevalência das alterações eletrocardiográficas, entre os indivíduos soro reagentes e os não reagentes, em todos os estados, exceção feita para o de Alagoas. O cálculo do gradiente mostrou-se maior no Estado do Paraná (42\%), seguido por Goiás (38,6\%), Ceará (32,3\%) Minas Gerais (31,9\%), Piauí (30,3\%), Paraíba (25,8\%), Pernambuco (22,3\%), Bahia (18,9\%), Sergipe (16,7\%), Rio Grande do Sul (9,9\%) e Alagoas (7,5\%). Em relação à distribuição das alterações eletrocardiográficas encontradas, nos onze estados analisados, verificou-se que as extrassístoles ventriculares, o bloqueio de grau avançado do ramo direito, o bloqueio da divisão ântero-superior do ramo esquerdo, a associação do bloqueio de grau avançado do ramo direito com o bloqueio da divisão ântero-superior do ramo esquerdo e alterações primárias do segmento ST e da onda T, mostraram diferença estatisticamente significativa, entre os indivíduos com sororreagentes e os não reagentes Além disto, estas alterações eletrocardiográficas foram as de maior prevalência no grupo dos indivíduos chagásicos.
\end{abstract}

Palavras-chaves: Doença de Chagas. Eletrocardiografia. Levantamentos epidemiológicos.

\begin{abstract}
In order to investigate the prevalence of chagasic heart disease in Brazil, a national electrocardiographic survey was carried out from 1977 to 1981. A total of 5,347 electrocardiograms (ECG) were performed and paired by age and gender. The results obtained in relation with the autochthonous cases, were distributed by Brazilian states, as follows: Rio Grande do Sul (1,078), Minas Gerais (760), Bahia (612), Paraná (400), Paraiba (340), Piauí (218), Sergipe (216), Goiás (176), Pernambuco (170), Ceará (136) and Alagoas 134. The higher proportions of altered ECGs among seropositive individuals were found in the States of Goiás (63.6\%), Minas Gerais (57.6\%), Ceará (57.3\%), Paraná (54.5\%), Piauí (53.2\%) and Paraiba (52.3\%). Among the control individuals, these proportions were respectively $25 \%, 25.7 \%, 25 \%, 12.5 \%, 22.9 \%$ and $26.5 \%$. A significant statistical difference of altered ECGs between positive and negative individuals was verified in all the States, with a single exception (Alagoas). The estimation of the gradient showed to be higher in Paraná State (42\%), followed by Goiás (38.6\%), Ceará (32.3\%), Minas Gerais (31.9\%), Piauí (30.3\%), Paraíba (25.8\%), Pernambuco (22.3\%), Bahia (18.9\%), Sergipe (16.7\%), Rio Grande do Sul (9.9\%) and Alagoas (7.5\%). Concerning the distribution of the electrocardiographical alterations found in the eleven states, the main alterations find among the seropositive group were: ventricular extrasystoles, complete right bundle branch block, left anterior fascicular block, the association of complete right bundle branch block with left anterior fascicular block and primary alterations of the ST segment and of the T wave. Furthermore, these ECG alterations were more prevalent in the group of infected individuals.
\end{abstract}

Key-words: Chagas's disease. Electrocardiography. Epidemiological surveys.

\section{INTRODUÇÃO}

Introduzido por Einthoven, em 1902, no Brasil, o eletrocardiograma (ECG) teve sua utilização progressivamente incorporada por cardiologistas e clínicos, especialmente, a partir dos anos 1920. Neste contexto, Carlos Chagas mais uma vez foi pioneiro realizando seus primeiros registros em casos de cardiopatia crônica da moléstia que vinha de descrever, tão eivada de quadros arrítmicos complexos como de desordens de propagação do estímulo e de fenômenos

*Professor Adjunto de Cardiologia da Universidade Federal do Triângulo Mineiro e Doutor em Medicina Tropical pela Universidade Federal do Triângulo Mineiro, Uberaba, MG. **Doutor em Medicina Tropical pela Universidade Federal do Triângulo Mineiro, Uberaba, MG. ${ }^{* * *}$ Pesquisador Titular da Fundação Oswaldo Cruz e Professor do Departamento de Clínica Médica da Faculdade de Medicina da Universidade Federal de Minas Gerais, Uberaba, MG. $\uparrow$ In memorian.

Endereço para correspondência: Dr. José Geraldo Ferreira Gonçalves. Rua Dr. Mozart Furtado Nunes 317/1000, Bairro Mercês, 38060-400 Uberaba, MG. e-mail: jgfg@terra.com.br fibróticos e inflamatórios no miocárdio. Já o fizera antes, empregando o polígrafo de Juqué e um eletrocardiógrafo primitivo registrando, entre 1911 e 1916, as extrassístoles ventriculares e a discincronia átrio-ventricular do BAV total ${ }^{1,2}$. O ECG foi depois empregado por ele e seu colega Eurico Villela, mais tarde com Evandro, logrando-se magníficas descrições e interpretações da cardiomiopatia chagásica já nos alvores da década de trinta ${ }^{3,4}$. Entendendo que a imensa maioria dos casos da tripanossomíase se encontrava na fase crônica, Chagas a esta se dedicou, buscando afirmar-lhe o diagnóstico e caracterizar a forma cardíaca, que sabia ser o quadro clínico mais importante da nova doença, com elevada incidência e impacto médico-social nas áreas endêmicas. Não obstante a clareza e a precisão dessas pesquisas, somente na década de quarenta começou a ser definitivamente aceita a cardiopatia crônica chagásica no meio médico-científico latino-americano, mercê de três fatos fundamentais: A) o aperfeiçoamento da reação de fixação de complemento para o diagnóstico etiológico, altamente sensível e específico da protozoose; B) a utilização corrente e bem interpretada do eletrocardiograma 
em casos crônicos detectados no campo ou no ambiente médicohospitalar; C) os trabalhos iniciais sistematizados de investigação clínico-eletrocardiográfica em áreas endêmicas, principalmente realizados por Emmanuel Dias, Francisco Laranja cols ${ }^{5}$, Ramos cols $^{6}$, Pondé ${ }^{7}$ e Aristóteles Brasil ${ }^{8}$, entre outros. Revista a cardiopatia crônica por Dias e cols ${ }^{9}$ em 1945 , e por Laranja ${ }^{10}$ em 1949 é especialmente importante o trabalho clássico de Laranja, Dias, Nóbrega e Miranda na Circulation (1956), que marcou definitivamente a sistematização da cardiopatia crônica chagásica, destacando o ECG como elemento fundamental em sua avaliação ${ }^{11}$. Fora do Brasil, o emprego do ECG em doença de Chagas se registra principalmente na Argentina, com Mazza e cols, em $1941^{12}$ e Romaña e Cossio, em $1944^{13}$.

O presente trabalho destina-se a retomar e re-analisar o primeiro inquérito eletrocardiográfico levado a cabo no Brasil, a partir do final dos anos setenta, por Macedo e cols, na esteira do grande inquérito sorológico nacional que começou a ser realizado, em $1976^{14,15}$. Como antecedentes mais específicos com respeito a inquéritos eletrocardiográficos sobre a esquizotripanose, complementando a introdução acima, poder-se-ia sumariar, numa escala cronológica:

- Em 1944,Emmanuel Dias se dá conta da grande frequência de cardiopatias agudas e crônicas entre chagásicos de Bambuí, MG, e lá instala um velho eletrocardiógrafo de corda com apoio do Prof.Magalhães Gomes, que mais tarde lhe indicará o cardiologista Dr. Francisco da Silva Laranja (seu discípulo e ex-estagiário do Dr. Paul White) para agregar-se aos estudos da cardiopatia chagásica e aprofundar as análises eletrocardiográficas. Um primeiro trabalho pormenorizado com análises eletrocardiográficas em crônicos chagásicos sairá em $1945^{9}$. Nesta época, entrará na rotina de Bambuí a sorologia por RFC para diagnóstico da infecção crônica, sob responsabilidade do Prof. Júlio Muniz, também do Instituto Oswaldo $\mathrm{Cruz}^{16-18}$;

- Em 1946-1947, Dias e cols realizarão modelar inquérito sorológico e clínico eletrocardiográfico nas imediações de Bambuí, entre as estações ferroviárias de Garças de Minas e Campos Altos, examinando aleatoriamente centenas de indivíduos entre a população geral não selecionada. Verifica-se uma prevalência próxima a $40 \%$ de pessoas com sorologia positiva, observando-se nestas uma frequência significativamente maior de alterações eletrocardiográficas que entre as não infectadas ${ }^{19}$. Mais tarde, este trabalho será complementado com observações anatomopatológicas entre pessoas falecidas e maior aprofundamento da análise eletrocardiográfica, por Laranja e cols ${ }^{20}$;

- Em 1949, Jairo Ramos e cols estudarão clínica, sorológica e eletrocardiograficamente 500 indivíduos não- selecionados, residentes no noroeste de São Paulo (Cássia dos Coqueiros), chegando a conclusões semelhantes a Dias e Laranja sobre a predominância e especificidade das alterações eletrocardiográficas, em portadores da doença de Chagas crônica, relativamente aos não-infectados ${ }^{6}$;

- Entre 1953 e 1954, o grupo de Manguinhos irá aprimorar a metodologia e a análise epidemiológica em inquéritos sorológico-eletrocardiográficos, comparando indivíduos não-selecionados soropositivos e soronegativos de áreas endêmicas (Minas Gerais) e não-endêmicas (Baixada Fluminense, Rio de Janeiro), atestando de vez a importância do componente etiológico, exclusivamente chagásico, na gênese e nas características da cardiopatia chagásica ${ }^{21}$;
- Entre 1950 e 1960, uma série de estudos eletrocardiográfcos pontuais será levada a cabo em várias partes do Brasil (Minas Gerais, São Paulo, Pernambuco, Goiás, Bahia, Ceará e Rio Grande do Sul), da Argentina e da Venezuela, geralmente comparando indivíduos soropositivos e soronegativos em populações não-selecionadas ou em grupos especiais (como crianças, indivíduos internados em Hospitais, beneficiários de Institutos de Pensões, etc). Apesar da diversidade das amostras, sempre ficará patente a maior incidência de alterações eletrocardiográficas entre os soropositivos, ao lado de entre os infectados, do predomínio de alterações nos indivíduos de maior idade e, também, quase sempre, predominando as alterações no gênero masculino ${ }^{17,18,22}$;

- Em 1956, Brant e cols irão mostrar o que chamariam de matizes regionais da doença de Chagas, detectando uma menor prevalência de alterações eletrocardiográficas entre populações não-selecionadas do Rio Grande do Sul, em comparação com os achados de outras áreas endêmicas ${ }^{23}$;

- No exterior, entre outros inquéritos pioneiros, ressalta o trabalho de Rosembaum e Cerisola, em 1956-57, que analisaram sorológica e eletrocardiograficamente uma população não-selecionada do norte de Córdoba e sul de Santiago del Estero (Argentina), zona de alta endemicidade da tripanossomíase, logrando resultados semelhantes aos de Dias, Laranja e Ramos, ressaltando a alta especificidade do BCRD na cardiopatia chagásica crônica e projetando, para seu país, cerca de $20 \%$ de cardiopatas crônicos entre os indivíduos infectados pelo Trypanosoma cruzi;

- Em 1960, 1970 e 1971, a Organização Mundial da Saúde realizará três reuniões importantes de especialistas (duas em Washington e uma em Caracas), objetivando um melhor conhecimento sobre a doença e seu controle, nas quais enfatizam a necessidade imperiosa de conhecerse a morbidade nos diferentes países endêmicos, como argumento para seu controle, enfatizando os inquéritos sorológicos e eletrocardiográficos como estratégia fundamental; na terceira reunião, mais enfaticamente, propõe estudos clínicos e eletrocardiográficos padronizados, para estudos pontuais e populacionais ${ }^{24-26}$;

- Em 1965-1966, Puigbó e cols estudarão duas comunidades rurais na Venezuela (Belen e Eneal), combinando estudos sorológicos e eletrocardiográficos, dos quais concluem pelo maior acometimento cardíaco entre os infectados e pelo caráter evolutivo da cardiopatia chagásica, em quatro anos de seguimento ${ }^{27}$;

- Em 1967, Cichero e cols estudaram convocados ao serviço militar em Córdoba, Argentina, sendo 2.878 soropositivos e 1.143 soronegativos, encontrando $34,7 \%$ de ECGs normais entre os primeiros e $87,5 \%$, entre os últimos. A incidência de bloqueio completo do ramo direito e extrassístoles ventriculares foram respectivamente de 29,5\% contra $1,7 \%$, e $5,9 \%$ contra $2,6 \%$. Na mesma região, estudando os achados eletrocardiográficos em população geral infectada, (2.622 pessoas), encontraram uma progressão de traçados alterados conforme a idade, oscilando de 1,9\% (5-9 anos) a $4,8 \%$ (10-19 anos ) a 8,8\% (30-39 anos), a 20,2\% (40-49 anos) a $27,7 \%$ (60 anos e mais) $)^{28}$; 
- Em 1968, Rose e Blackburn editarão a monografia sobre metodologias de inquéritos em doenças cardiovasculares, um marco fundamental na padronização de estudos clínico populacionais ${ }^{29}$;

- Em 1974, no Brasil, o Conselho Nacional de Desenvolvimento Científico e Tecnológico (CNPq) editará um opúsculo técnico sobre estudos longitudinais, mencionando o ECG como ferramenta fundamental nas avaliações de morbidade ${ }^{30}$;

- Em 1975, numa reunião em Brasília sobre diferenças regionais em doença de Chagas ${ }^{31}$, os Profs. Guilherme Rodrigues da Silva e José Rodrigues Coura propõem formalmente (p. 162 a 166) a realização sistemática de inquéritos sorológicos padronizados em diferentes áreas, elegendo-se o eletocardiograma como o mais objetivo marcador da cardiopatia crônica chagásica, sendo suas alterações muito mais precoces do que as manifestações clínicas e radiológicas;

- Em 1984, finalizado o inquérito sorológico nacional e já em andamento o $1^{\circ}$ inquérito eletrocardiográfico, o Dr Armênio Guimarães, num curso em Belo Horizonte, enfatizou o valor dos inquéritos eletrocardiográficos para estudos de morbidade em doença de Chagas, destacando a necessidade de padronização em sua aplicação e leitura, á época propondo como referência o código de Minesotta ${ }^{32}$;

- Em 1985, o Programa Salud Humana, da Argentina, reuniu eminentes cardiologistas e confeccionou um Manual de Eletrocardiografia apropriado para a cardiopatia chagásica, com ampla difusão naquele país, sendo esta a primeira tentativa de normatização da leitura e nomenclatura eletrocardiográfica com vistas a uma mais ampla padronização ${ }^{33}$;

- Em 1986, num encontro da Organização Mundial da Saúde (OMS), em Belo Horizonte, sobre doença de Chagas, enfatizou-se a necessidade de estandardizar protocolos para análise clínica, radiológica, eletrocardiográfica e laboratorial em estudos epidemiológicos para o diagnóstico da referida patologia. Em abril de 1998, sob os auspícios da OMS, um grupo de seis cardiologistas, de cinco países (Júlio O. Lázzari da Argentina, Alfredo Romero da Bolívia, Vanize Macêdo e Anis Rassi do Brasil, Rafael Chávez Dominguez do México e Otto Hernández Pieretti da Venezuela) reuniram-se em Buenos Aires e estandardizaram um método de leitura de eletrocardiograma para estudos epidemiológicos em doença de Chagas $^{34}$. Este quarto método para a leitura eletrocardiográfica, e terceiro para estudos longitudinais, ficou conhecido com Método de Buenos Aires, e foi publicado na Revista Panamericana de Salud Pública ${ }^{34}$.

\section{MÉTODOS}

\section{Pacientes e métodos}

Entre 1975 e 1981, por iniciativa do CNPq, o Ministério da Saúde do Brasil, através da Superintendência das Campanhas (SUCAM), e com o auxílio de várias instituições, realizou um inquérito sorológico para doença de Chagas, tendo por objetivo estimar a prevalência e a distribuição geográfica da doença de Chagas no território nacional. O referido inquérito teve por base a reação de imunofluorescência, realizada em sangue colhido em papel de filtro, em zonas rurais, sendo que os exames foram realizados em laboratórios de referência, sob a coordenação do Dr. Mário Camargo ${ }^{14}$.

Em um braço do referido inquérito, entre 1977 e 1981, com o intuito de avaliar a prevalência da cardiomiopatia chagásica no Brasil, realizou-se um inquérito eletrocardiográfico.

Em onze estados do Brasil, ou seja, Minas Gerais (MG), Rio Grande do Sul (RS), Bahia (BA), Goiás (GO), Piauí (PI), Paraíba (PB), Pernambuco (PE), Sergipe (SE), Ceará (CE), Alagoas (AL) e Paraná (PR), foram escolhidos alguns municípios para o estudo eletrocardiográfico de indivíduos autóctones. Uma amostra significativa de pessoas com soro reagente foi pareada, de acordo com idade e gênero, com controles (soros não-reagentes). A relação dos nomes e endereços foi encaminhada ao Núcleo de Medicina Tropical e Nutrição da Universidade de Brasília ${ }^{14}$.

Com o auxílio da SUCAM regional e das prefeituras de cada município, um técnico localizava as diversas vilas ou fazendas e dos indivíduos selecionados, na própria residência foi realizado um eletrocardiograma em repouso de 12 derivações ${ }^{14}$.

Durante todo o estudo, foi utilizado um eletrocardiógrafo portátil transistorizado. Todos os traçados foram realizados pela mesma técnica, seguindo-se as normas de Metodologia de Estudos Longitudinais (CNPq, 1974). Em algumas localidades, por não haver energia elétrica, o eletrocardiógrafo era ligado à bateria do veículo que conduzia a técnica (V Macêdo: comunicação pessoal, 2002). Os eletrocardiogramas foram enviados ao Núcleo de Medicina Tropical e Nutrição da Universidade de Brasília (V Macêdo: comunicação pessoal, 2002).

Em 2002, de acordo com os Professores Aluízio Prata, Vanize Macêdo e João Carlos Pinto Dias, durante a XVIII Reunião Anual de Pesquisa Aplicada em Doença de Chagas e VI Reunião Anual de Pesquisa Aplicada em Leishmanioses, na Cidade de Uberaba (MG), decidiu-se realizar a leitura destes eletrocardiogramas pelo Código de Buenos Aires ${ }^{34}$. O referido código mostrou-se superior para a leitura de eletrocardiogramas em portadores de cardiopatia chagásica crônica ${ }^{35}$, quando comparado com a nomenclatura da New York Heart Association ${ }^{36}$ e com o Código de Minnesota adaptado ${ }^{37}$.

Os eletrocardiogramas foram enviados para a disciplina de Medicina Tropical e Infectologia da Universidade Federal do Triângulo Mineiro em fevereiro de 2003. A leitura dos mesmos foi realizada no período compreendido entre 2003 e 2007, pelo mesmo observador, sem que soubesse, no momento da leitura, o resultado da sorologia. Para a referida leitura foi utilizado um compasso, uma lupa, uma régua própria para a contagem da frequência cardíaca e uma calculadora da marca Casio, modelo Time Face QA-700. Os dados encontrados foram transpostos para um banco de dados. Após a conclusão da leitura, identificouse o resultado da sorologia (reagente ou não-reagente). Os eletrocardiogramas foram então, pareados de acordo com a idade e gênero dos indivíduos a que pertenciam.

\section{Análise estatística}

Nos onze estados avaliados, para comparação entre os indivíduos soro reagentes e os não reagentes, quanto às alterações eletrocardiográficas, utilizamos os seguintes métodos estatísticos: I) Teste do Qui ${ }^{2}$ corrigido de Yates (pacote estatístico - Epi Info version 3.3.2, 2005); II) odds ratio (OR) e seu intervalo de confiança 95\% (nível de confiança de 5\%, com poder estatístico de $80 \%$ ) (pacote estatístico - Epi Info - version 3.3.3, 2005). 


\section{RESULTADOS}

Inicialmente, 7.784 pessoas foram selecionadas para serem submetidas ao eletrocardiograma. No final do trabalho foram realizados 5.347 eletrocardiogramas. Dois mil quatrocentos e trinta e sete eletrocardiogramas não puderam ser feitos, ou porque as pessoas não foram novamente encontradas, ou porque mudaram da localidade de origem. Dos 5.347 eletrocardiogramas, vários não puderam ser lidos. A maioria porque o registro foi apagado pelo tempo, outros porque derivações precordiais não foram realizadas e alguns pela alta incidência de interferência no traçado.

Dos que puderam ser lidos, e após o pareamento dos indivíduos, segundo a idade e gênero, a distribuição dos eletrocardiogramas, em cada estado, ficou assim constituída: Rio Grande do Sul (1.078), Minas Gerais (760), Bahia (612), Paraná (400), Paraíba (340), Piauí (218), Sergipe (216), Goiás (176), Pernambuco (170), Ceará (136) e Alagoas (134) (Tabela 1).
TABELA 1 - Distribuição dos eletrocardiogramas, segundo sorologia para Trypanosoma cruzi e pareamento dos indivíduos por idade e gênero, nos onze estados.

\begin{tabular}{lcccc}
\hline & \multicolumn{4}{c}{ Indivíduos } \\
\cline { 2 - 5 } & Soro & Soro & \multicolumn{2}{c}{ Total } \\
\cline { 3 - 5 } Estados & não-reagente & reagente & $\mathrm{n}^{\circ}$ & $\%$ \\
\hline Rio Grande Sul & 539 & 539 & 1.078 & 25,4 \\
Minas Gerais & 380 & 380 & 760 & 17,9 \\
Bahia & 306 & 306 & 612 & 14,5 \\
Paraná & 200 & 200 & 400 & 9,4 \\
Paraíba & 170 & 170 & 340 & 8,0 \\
Piauí & 109 & 109 & 218 & 5,1 \\
Sergipe & 108 & 108 & 216 & 5,1 \\
Goiás & 88 & 88 & 176 & 4,2 \\
Pernambuco & 85 & 85 & 170 & 4,0 \\
Ceará & 68 & 68 & 136 & 3,2 \\
Alagoas & 67 & 67 & 134 & 3,2 \\
\hline Total & $\mathbf{2 . 1 2 0}$ & $\mathbf{2 . 1 2 0}$ & $\mathbf{4 . 2 4 0}$ & $\mathbf{1 0 0 , 0}$ \\
\hline
\end{tabular}

A Tabela 2 evidencia a distribuição dos eletrocardiogramas, segundo a sorologia e gênero das pessoas em cada estado. Verificamos uma maior prevalência para o gênero feminino, tanto para os indivíduos com soro não-reagente como para aqueles com soros reagentes, em todos os estados, com exceção do Estado do Ceará (Tabela 2).

A Tabela 3 mostra a distribuição dos eletrocardiogramas, segundo a sorologia e as faixas etárias dos indivíduos nos 11 estados. Não encontramos pessoas, tanto com soros não-reagentes, quanto reagentes, na faixa etária de 0 a 14 anos. Observamos que a maior prevalência das mesmas ocorreu entre a faixa etária de 20 e 49 anos (Tabela 3).

A Tabela 4 evidencia a prevalência das alterações eletrocardiográficas entre os indivíduos com soro reagente e os não-reagente para T. cruzi, o cálculo do odds ratio e p, em cada estado pesquisado. Os maiores percentuais de alterações eletrocardiográficas entre os indivíduos com soro reagente foram
TABELA 2 - Distribuição dos eletrocardiogramas, segundo a sorologia para Trypanosoma cruzi e gênero dos indivíduos, nos onze estados.

\begin{tabular}{|c|c|c|c|c|c|c|c|c|}
\hline \multirow[b]{4}{*}{ Estados } & \multicolumn{8}{|c|}{ Indivíduos } \\
\hline & \multicolumn{4}{|c|}{$\begin{array}{l}\text { Soro não-reagente } \\
\text { gênero }\end{array}$} & \multicolumn{4}{|c|}{$\begin{array}{l}\text { Soro reagente } \\
\text { gênero }\end{array}$} \\
\hline & \multicolumn{2}{|c|}{ masculino } & \multicolumn{2}{|c|}{ feminino } & \multicolumn{2}{|c|}{ masculino } & \multicolumn{2}{|c|}{ feminino } \\
\hline & $\mathrm{n}^{0}$ & $\%$ & $\mathrm{n}^{\circ}$ & $\%$ & $\mathrm{n}^{\circ}$ & $\%$ & $\mathrm{n}^{\circ}$ & $\%$ \\
\hline Rio Grande do Sul & 230 & 42,7 & 309 & 57,3 & 230 & 42,7 & 309 & 57,3 \\
\hline Minas Gerais & 160 & 42,1 & 220 & 57,9 & 160 & 42,1 & 220 & 57,9 \\
\hline Bahia & 104 & 34,0 & 202 & 66,0 & 104 & 34,0 & 202 & 66,0 \\
\hline Paraná & 87 & 43,5 & 113 & 56,5 & 87 & 43,5 & 113 & 56,5 \\
\hline Paraíba & 62 & 36,5 & 108 & 63,5 & 62 & 36,5 & 108 & 63,5 \\
\hline Piauí & 39 & 35,8 & 70 & 64,2 & 39 & 35,8 & 70 & 64,2 \\
\hline Sergipe & 31 & 28,7 & 77 & 71,3 & 31 & 28,7 & 77 & 71,3 \\
\hline Goiás & 32 & 36,4 & 56 & 63,6 & 32 & 36,4 & 56 & 63,6 \\
\hline Pernambuco & 26 & 30,6 & 59 & 69,4 & 26 & 30,6 & 59 & 69,4 \\
\hline Ceará & 47 & 69,1 & 21 & 30,9 & 47 & 69,1 & 21 & 30,9 \\
\hline Alagoas & 23 & 34,3 & 44 & 65,7 & 23 & 34,3 & 44 & 65,7 \\
\hline Total & 841 & & 1.279 & & 841 & & 1.279 & \\
\hline
\end{tabular}

TABELA 3 - Distribuição dos eletrocardiogramas, segundo a sorologia para Trypanosoma cruzi e faixas etárias dos indivíduos, por estado.

\begin{tabular}{|c|c|c|c|c|c|c|c|c|c|c|c|c|c|c|c|c|c|c|c|c|c|c|c|c|c|c|}
\hline \multirow[b]{3}{*}{ Estados } & \multicolumn{26}{|c|}{ Faixas etárias (anos) } \\
\hline & \multicolumn{2}{|c|}{$0-4$} & \multicolumn{2}{|c|}{$5-9$} & \multicolumn{2}{|c|}{$10-14$} & \multicolumn{2}{|c|}{ 15-19 } & \multicolumn{2}{|c|}{$20-24$} & \multicolumn{2}{|c|}{$25-29$} & \multicolumn{2}{|c|}{$30-34$} & \multicolumn{2}{|c|}{$35-39$} & \multicolumn{2}{|c|}{$40-44$} & \multicolumn{2}{|c|}{$45-49$} & \multicolumn{2}{|c|}{$50-54$} & \multicolumn{2}{|c|}{$55-59$} & \multicolumn{2}{|c|}{$>59$} \\
\hline & $\bar{C}$ & $\overline{\mathrm{D}}$ & $\bar{C}$ & $\overline{\mathrm{D}}$ & $\bar{C}$ & $\overline{\mathrm{D}}$ & $\mathrm{C}$ & $\bar{D}$ & $\mathrm{C}$ & $\overline{\mathrm{D}}$ & $\mathrm{C}$ & $\overline{\mathrm{D}}$ & $\mathrm{C}$ & $\overline{\mathrm{D}}$ & $\bar{C}$ & $\overline{\mathrm{D}}$ & $\mathrm{C}$ & $\overline{\mathrm{D}}$ & $\mathrm{C}$ & $\overline{\mathrm{D}}$ & $\mathrm{C}$ & $\overline{\mathrm{D}}$ & $\bar{C}$ & $\overline{\mathrm{D}}$ & $\bar{C}$ & $\overline{\mathrm{D}}$ \\
\hline Minas Gerais & 0 & 0 & 0 & 0 & 0 & 0 & 15 & 17 & 32 & 33 & 68 & 65 & 48 & 48 & 60 & 60 & 46 & 46 & 40 & 41 & 31 & 31 & 26 & 24 & 14 & 15 \\
\hline Rio Grande do Sul & 0 & 0 & 0 & 0 & 0 & 0 & 37 & 37 & 76 & 72 & 52 & 56 & 51 & 50 & 53 & 51 & 63 & 69 & 67 & 65 & 66 & 58 & 53 & 60 & 21 & 21 \\
\hline Bahia & 0 & 0 & 0 & 0 & 0 & 0 & 68 & 65 & 52 & 53 & 48 & 43 & 32 & 36 & 38 & 36 & 18 & 23 & 21 & 19 & 16 & 18 & 8 & 9 & 5 & 4 \\
\hline Goiás & 0 & 0 & 0 & 0 & 0 & 0 & 19 & 18 & 15 & 17 & 18 & 16 & 6 & 7 & 5 & 5 & 8 & 8 & 6 & 5 & 4 & 4 & 6 & 5 & 1 & 3 \\
\hline Piauí & 0 & 0 & 0 & 0 & 0 & 0 & 8 & 5 & 17 & 14 & 10 & 16 & 16 & 15 & 11 & 11 & 14 & 18 & 15 & 10 & 8 & 6 & 9 & 9 & 1 & 5 \\
\hline Paraíba & 0 & 0 & 0 & 0 & 0 & 0 & 5 & 4 & 10 & 15 & 18 & 13 & 20 & 22 & 19 & 16 & 19 & 21 & 19 & 18 & 21 & 24 & 19 & 17 & 20 & 20 \\
\hline Pernambuco & 0 & 0 & 0 & 0 & 0 & 0 & 6 & 6 & 9 & 7 & 8 & 9 & 16 & 14 & 15 & 17 & 12 & 14 & 5 & 4 & 8 & 8 & 4 & 4 & 2 & 2 \\
\hline Sergipe & 0 & 0 & 0 & 0 & 0 & 0 & 11 & 10 & 11 & 11 & 14 & 13 & 16 & 18 & 14 & 10 & 10 & 12 & 12 & 13 & 7 & 9 & 8 & 10 & 5 & 2 \\
\hline Ceará & 0 & 0 & 0 & 0 & 0 & 0 & 1 & 1 & 7 & 9 & 8 & 9 & 9 & 7 & 8 & 7 & 7 & 6 & 6 & 7 & 5 & 7 & 9 & 9 & 8 & 6 \\
\hline Alagoas & 0 & 0 & 0 & 0 & 0 & 0 & 9 & 11 & 18 & 15 & 13 & 16 & 9 & 9 & 6 & 4 & 3 & 3 & 4 & 4 & 2 & 1 & 1 & 3 & 2 & 1 \\
\hline Paraná & 0 & 0 & 0 & 0 & 0 & 0 & 22 & 24 & 22 & 25 & 31 & 27 & 28 & 27 & 23 & 23 & 24 & 27 & 20 & 19 & 10 & 11 & 18 & 15 & 2 & 2 \\
\hline Total & 0 & 0 & 0 & 0 & 0 & 0 & 201 & 198 & 269 & 271 & 288 & 283 & 251 & 253 & 252 & 240 & 224 & 247 & 215 & 205 & 178 & 177 & 161 & 165 & 81 & 81 \\
\hline
\end{tabular}


TABELA 4 - Prevalência das alterações eletrocardiográficas, por estado, segundo a sorologia para Trypanosoma cruzi, odds ratio e p.

\begin{tabular}{|c|c|c|c|c|c|c|c|c|}
\hline \multirow[b]{3}{*}{ Estados } & \multirow{3}{*}{$\begin{array}{c}\text { ECG } \\
\mathrm{n}^{\circ}\end{array}$} & \multirow{2}{*}{\multicolumn{2}{|c|}{$\begin{array}{c}\text { Indivíduos soro } \\
\text { reagente } \\
\text { ECG com alterações }\end{array}$}} & \multirow{3}{*}{$\begin{array}{c}\text { ECG } \\
\mathrm{n}^{\circ}\end{array}$} & \multirow{2}{*}{\multicolumn{2}{|c|}{$\begin{array}{c}\text { Indivíduos soro } \\
\text { não reagente } \\
\text { ECG com alterações }\end{array}$}} & \multirow[b]{3}{*}{ Odds ratio } & \multirow[b]{3}{*}{$\mathrm{p}$} \\
\hline & & & & & & & & \\
\hline & & $\mathrm{n}^{\circ}$ & $\%$ & & $\mathrm{n}^{\circ}$ & $\%$ & & \\
\hline Rio Grande do Sul & 539 & 160 & 29,7 & 539 & 107 & 19,8 & $1,70(1,27-2,28)$ & $<0,001$ \\
\hline Minas Gerais & 380 & 219 & 57,6 & 380 & 98 & 25,7 & $3,91(2,85-5,39)$ & $<0,001$ \\
\hline Bahia & 306 & 121 & 39,5 & 306 & 63 & 20,6 & $2,52(1,73-3,67)$ & $<0,001$ \\
\hline Paraná & 200 & 109 & 54,5 & 200 & 25 & 12,5 & $8,38(4,93-14,33)$ & $<0,001$ \\
\hline Paraíba & 170 & 89 & 52,3 & 170 & 45 & 26,5 & $3,05(1,89-4,94)$ & $<0,001$ \\
\hline Piauí & 109 & 58 & 53,2 & 109 & 25 & 22,9 & $3,82(2,05-7,16)$ & $<0,001$ \\
\hline Sergipe & 108 & 50 & 46,3 & 108 & 32 & 29,6 & $2,05(1,13-3,73)$ & $=0,01$ \\
\hline Goiás & 88 & 56 & 63,6 & 88 & 22 & 25 & $5,25(2,61-10,62)$ & $<0,001$ \\
\hline Pernambuco & 85 & 37 & 43,5 & 85 & 18 & 21,2 & $2,87(1,39-5,97)$ & 0,003 \\
\hline Ceará & 68 & 39 & 57,3 & 68 & 17 & 25 & $4,03(1,83-8,97)$ & $<0,001$ \\
\hline Alagoas & 67 & 26 & 38,8 & 67 & 21 & 31,3 & $1,39(0,64-3,02)$ & $=0,46$ \\
\hline
\end{tabular}

encontrados nos Estados de Goiás (63,6\%), Minas Gerais (57,6\%), Ceará (57,3\%), Paraná (54,5\%), Piauí (53,2\%) e Paraíba (52,3\%). Entre os controles, nestes estados, a prevalência das alterações eletrocardiográficas foi de 25\%, 25,7\%,25\%, 12,5\%, 22,9\% e 26,5\% respectivamente (Tabela 4 ).

Em relação à análise do odds ratio, verificamos que no Estado do Paraná os indivíduos com sorologia positiva para $T$ cruzi tiveram $8,38($ IC95\% $=4,93-14,33)$ vezes mais chance de apresentarem alterações eletrocardiográficas quando comparados com aqueles com sorologia negativa. O cálculo de $p$ foi estatisticamente significativo ( $p<0,001)$ (Tabela 4). Quanto aos demais estados, encontramosaseguinte ordem decrescente segundo resultados do odds ratio: Goiás [5,25 (IC95\%=2,610,62)], Ceará $[4,03($ IC95\%=1,83-8,97)], Minas Gerais [3,91 (IC95\%=2,85-5,39)], Piauí $[3,82$ (IC95\%=2,05-7,16)], Paraíba $[3,05$ (IC95\%=1,89-4,94)], Pernambuco $[2,87($ IC95\%=1,39-5,97)], Bahia $[2,52$ (IC 95\%=1,73-3,67)], Sergipe $[2,05$ (IC95\%=1,13-3,73)], Rio Grande do Sul $[1,70$ (IC95\%=1,27-2,28)] e Alagoas [1,39 (IC95\%=0,64-3,02)] (Tabela 4). Alagoas figurou como o único estado onde o cálculo de p não se mostrou estatisticamente significativo $(p=0,46)$ (Tabela 4).

Utilizando-se ainda os dados encontrados na Tabela 4, pudemos calcular o gradiente (diferença entre as alterações encontradas nos eletrocardiogramas dos indivíduos soro reagentes e os não-reagentes). Verificamos que o mesmo teve seu maior valor no Estado do Paraná (42\%), seguido por Goiás $(38,6 \%)$, Ceará (32,3\%), Minas Gerais (31,9\%),
TABELA 5 - Distribuição das alterações eletrocardiográficas encontradas, nos estados investigados, segundo sorologia para Trypanosoma cruzi, odds ratio e p.

\begin{tabular}{|c|c|c|c|c|c|c|}
\hline \multirow[b]{3}{*}{ Alterações do ECG } & \multicolumn{4}{|c|}{ Sorologia para Tripanosoma cruzi } & \multirow[b]{3}{*}{ Odds ratio } & \multirow[b]{3}{*}{$\mathrm{p}$} \\
\hline & \multicolumn{2}{|c|}{ Soro não reagente } & \multicolumn{2}{|c|}{ Soro reagente } & & \\
\hline & $\mathrm{n}^{\circ}$ & $\%$ & $\mathrm{n}^{\circ}$ & $\%$ & & \\
\hline BAV primeiro grau & 12 & 0,6 & 21 & 1,0 & $1,76($ IC95\%=0,82-3,80) & $=0,16$ \\
\hline Ritmo juncional & 4 & 0,2 & 3 & 0,1 & $0,75($ IC95\%=0,13-3,95) & $=0,50$ \\
\hline Ritmo migratório & 1 & 0,05 & 3 & 0,1 & $3,00($ IC95\% $=0,28-74,92)$ & $=0,31$ \\
\hline FA & 3 & 0,1 & 4 & 0,2 & $1,33($ IC95\%=0,25-7,48) & $=0,50$ \\
\hline ESSV & 20 & 0,9 & 26 & 1,2 & $1,30($ IC95\%=0,70-2,43) & $=0,45$ \\
\hline ESV & 42 & 2,0 & 91 & 4,3 & $2,22($ IC95\%=1,51-3,27) & $<0,001$ \\
\hline BGNARD & 31 & 1,5 & 40 & 1,9 & $1,30($ IC95\%=0,79-2,13) & $=0,33$ \\
\hline BGARD & 16 & 0,7 & 78 & 3,7 & $5,02($ IC95\%=2,85-8,97) & $<0,001$ \\
\hline BDASE & 35 & 1,6 & 63 & 3,0 & $1,82(\mathrm{IC} 95 \%=1,18-2,83)$ & $=0,005$ \\
\hline BGARD+BDASE & 17 & 0,8 & 62 & 2,9 & $3,73($ IC95\%=2,12-6,64) & $<0,001$ \\
\hline BGARE & 6 & 0,3 & 10 & 0,5 & $1,67($ IC95\% $=0,56-5,16)$ & $=0,45$ \\
\hline BDPIE & 1 & 0,05 & 2 & 0,1 & $2,00($ IC95\% $=0,14-55,70)$ & $=0,50$ \\
\hline Alt. Q & 100 & 4,7 & 208 & 9,8 & 2,20 (IC95\%=1,71-2,83) & $<0,001$ \\
\hline Alt. ST e T & 139 & 6,5 & 283 & 13,3 & $2,20($ IC95\%=1,77-2,73) & $<0,001$ \\
\hline SVE & 34 & 1,6 & 45 & 2,1 & $1,33($ IC95\%=0,83-2,14) & $=0,25$ \\
\hline SVD & 7 & 0,3 & 9 & 0,4 & $1,29($ IC95\% $=0,44-3,83)$ & $=0,80$ \\
\hline Baixa voltagem $\mathrm{QRS}$ & 0 & 0,0 & 3 & 0,1 & Indefinido & $=0,12$ \\
\hline Bradicardia siunusal & 5 & 0,2 & 13 & 0,6 & $2,61($ IC $95 \%=0,87-8,38)$ & $=0,09$ \\
\hline
\end{tabular}

ECG: eletrocardiograma, BAV: bloqueio átrio-ventricular, FA: fibrilação atrial, ESSV: extrassístoles supraventriculares, ESV: extrassístoles ventriculares, BGNARD: bloqueio de grau não avançado do ramo direito, BGARD: bloqueio de grau avançado do ramo direito, BDASE: bloqueio da divisão ântero-superior do ramo esquerdo, BGARE: bloqueio de grau avançado do ramo esquerdo, BDPIE: bloqueio da divisão póstero-inferior do ramo esquerdo, SVE: sobrecarga ventricular esquerda, SVD: sobrecarga ventricular direita, IC: intervalo de confiança. 
vezes mais chance de apresentarem esta alteração no eletrocardiograma, quando comparadas com as de soro não reagente, havendo diferença estatisticamente significativa ( $p<0,001)$ (Tabela 5). Seguem por ordem de importância: bloqueio de grau avançado do ramo direito associado ao bloqueio da divisão ântero-superior do ramo esquerdo [3,73 (IC95\%=2,12-6,64); $p<0,001]$, extrassístoles ventriculares [2,22 (IC95\%=1,51-3,27); $p<0,001]$, alterações do segmento ST e da onda T [2,20 (IC95\%=1,77-2,73); $p<0,001]$, alterações da onda Q $[2,20$ (IC95\%=1,71-2,83); $\mathrm{p}<0,001]$ e bloqueio da divisão ântero-superior do ramo esquerdo [1,82 (IC95\%=1,18-2,83); $p=0,005]$. As demais alterações não mostraram diferença estatisticamente significativa (Tabela 5).

\section{DISCUSSÃO}

No Brasil, o padrão das formas clínicas da doença de Chagas mostra diferenças regionais tanto na gravidade da doença como na predominância da localização das lesões no ser humano ${ }^{38}$. A interpretação das causas dessas variações é bastante complexa. Entretanto, determinados fatores, alguns ligados ao indivíduo parasitado (estado imunológico, estado nutricional, fator genético, esforço físico), outros relacionados ao parasito (diferentes cepas do T. cruzi, intensidade do parasitismo, reinfecções), exercem influência importante para o achado destas discrepâncias regionais.

Neste inquérito eletrocardiográfico, observamos que houve diferença estatisticamente significativa, em relação à prevalência das alterações eletrocardiográficas, entre os indivíduos soro reagentes e os não-reagentes, em todos os estados, exceção feita para o de Alagoas (Tabela 4). Esta maior prevalência de alterações eletrocardiográficas nos indivíduos chagásicos quando comparados com os não-chagásicos, é corroborada por outros autores ${ }^{39-42}$. No Estado do Rio Grande do Sul, a prevalência das alterações eletrocardiográficas nos pacientes com soro reagentes foi de $29,7 \%$, enquanto entre os controles foi de 19,8\% (Tabela 4). O cálculo do odds ratio mostrou que os indivíduos com soro reagentes tiveram 1,70 (IC95\%=1,27-2,28) vezes mais chance de terem alterações eletrocardiográficas quando comparados com os de soro não reagentes, havendo diferença estatisticamente significativa $(p<0,001)$ (Tabela 4). Estes dados contrastam com os achados de Brant $^{23} \mathrm{e}$ Baruffa ${ }^{43}$, que não encontraram diferença significativa entre as prevalências de cardiopatia, estudando chagásicos e não-chagásicos no Rio Grande do Sul. Contrasta também com os achados das informações preliminares sobre o inquérito Nacional, onde Macêdo e colaboradores não encontraram diferença estatisticamente significativa entre os indivíduos soro reagentes e os não reagentes do Rio Grande do Sul $(p=0,18)^{14}$. Porém, na época da publicação destas informações, a realização dos eletrocardiogramas ainda não estava concluída, e também, não havia sido feito o pareamento, quanto à idade e gênero, na comparação dos mesmos ${ }^{14}$.

Quanto à distribuição das alterações eletrocardiográficas encontradas, nos onze estados analisados, verificamos que as extrassístoles ventriculares, o bloqueio de grau avançado do ramo direito, o bloqueio da divisão ântero-superior do ramo esquerdo, a associação do bloqueio de grau avançado do ramo direito com o bloqueio da divisão ântero-superior do ramo esquerdo e alterações primárias do segmento ST e da onda $\mathrm{T}$, mostraram diferença estatisticamente significativa, entre os indivíduos com soro reagente e os não reagentes (Tabela 5). Além disto, estas alterações eletrocardiográficas foram as mais frequentes encontradas no grupo dos indivíduos chagásicos (Tabela 5). Estes achados são corroborados por outros autores $22,35,40,42,44-46$.

Observamos uma maior prevalência das alterações da onda $\mathrm{Q}$, do segmento ST e da onda $\mathrm{T}$, tanto para os indivíduos chagásicos quanto para os controles, quando comparadas com as demais alterações eletrocardiográficas encontradas (Tabela 5). Provavelmente, este fato pode ser explicado porque alguns eletrocardiogramas apresentavam overdamping e/ou overshooting em seus standards. A presença dos mesmos favorece o aparecimento de alterações do segmento ST e da onda Q (Josef Feher: comunicação pessoal, 1980).

\section{REFERÊNCIAS}

1. Chagas CJR. O mal de Chagas. Arch Soc Med Cirurg 1912; 3:34-66.

2. Chagas CRJ. Aspectos clínicos y anátomo-patológicos de la tripanosomiasis americana. La Prensa Médica Argentina 1916; 15:153-158.

3. Chagas CRJ, Villela EA. Forma cardíaca da Tripanosomíase Americana. Mem Inst Oswaldo Cruz 1922; 14:5-61.

4. Chagas E. Estudo electro-cardiográfico na forma cardíaca da Tripanosomíase Americana. [Tese]. [Rio de Janeiro]: Faculdade Nacional de Medicina; 1930.

5. Dias E. Profilaxia da doença de Chagas. Resumo das principais atividades do Centro de Estudos do Instituto Oswaldo Cruz em Bambuí, Minas Gerais, durante dois anos. Brazil Med 1946; 60:161-163.

6. Ramos-Jr J, Borges S, D’Ávila M, Freitas JLP, Lindemberg S, Fonseca E. Estudo clínico e epidemiológico da moléstia de Chagas em 500 indivíduos residentes no distrito de Cássia dos Coqueiros (S. Paulo, Brasil). Arq Bras Cardiol 1949; 2:115-134.

7. Pondé A. A cardiopatia crônica da doença de Carlos Chagas. Arq Bras Cardiol 1948; 35:27-70.

8. Brasil A. Forma cardíaca crônica da doença de Chagas. Hospital 1946; 29:199-224.

9. Dias E, Laranja FS, Nóbrega GC. Doença de Chagas. Mem Inst Oswaldo Cruz 1945; 43:495-582.

10. Laranja FS. Evolução dos conhecimentos sobre a cardiopatia da doença de Chagas. Revisão crítica da literatura. Mem Inst Oswaldo Cruz 1949; 47:607-649.

11. Laranja FS, Dias E, Nóbrega GC, Miranda A. Chagas‘ Disease: a clinical, epidemiologic and pathologic study. Circulation 1956; XIV:1035-1060.

12. Mazza S, Myara S, Basso G, Basso R. Primer quinquenio de la investigación por la MEPRA de la Enfermedad de Chagas en la provincia de Mendoza. Mendoza: MEPRA; 1941.

13. Romaña C, Cossio F. Formas cardíacas de la enfermedad de Chagas. An Inst Med Reg (Tucumán) 1944; 1:9.

14. Macêdo VO, Prata A, Silva GR, Castilho E. Prevalência de alterações eletrocardiográficas em chagásicos. (Informações preliminares sobre o inquérito eletrocardiográfico nacional). Arq Bras Cardiol 1982; 38: 261-264.

15. Camargo ME, Silva GR, Castilho EA, Silveira AC. Inquérito sorológico da prevalência de infecção chagásica no Brasil, 1975/1980. Rev Inst Med Trop S Paulo 1984; 26:192-204.

16. Dias E. O Centro de Estudos e Profilaxia de Moléstia de Chagas, em Bambuí, Estado de Minas Gerais. Notícia histórica em homenagem ao professor Henrique Aragão. Mem Inst Oswaldo Cruz 1956; 54:309-357. 
17. Dias JCP. Doença de Chagas em Bambuí, Minas Gerais, Brasil. Estudo clínico-epidemiológico a partir da fase aguda, entre 1940 e 1982. [Tese]. [Belo Horizonte]: Universidade Federal de Minas Gerais; 1982.

18. Muniz J, Freitas G. Contribuição para o diagnóstico da doença de Chagas pelas reações de imunidade. 1. Estudo comparativo entre as reações de aglutinação e de fixação de complemento. Mem Inst Oswaldo Cruz 1944; 41:303-333.

19. Dias E, Laranja FS, Pellegrino J. Estudo sobre a importância social da doença de Chagas. I- Inquérito clínico-epidemiológico feito nas vizinhanças de Bambuí, oeste de Minas. Brazil Med 1948, 62:412-419.

20. Laranja FS, Dias E, Duarte E, Pellegrino J. Observações clínicas e epidemiológicas sobre a moléstia de Chagas no oeste de Minas Gerais. Hospital 1951; 40:945-956.

21. Dias E, Laranja FS, Nery Guimarães F, Brant TC. Estudo preliminar de inquéritos soro-eletrocardiográficos em populações não selecionadas de zonas não endêmicas e de zonas endêmicas de doença de Chagas. Ver Bras Malariol D Trop 1955; 5:205-212.

22. Storino R, Puigbó, JJ, Acquatella H, Madoery C, Esparza E, Mautner B, et al Estúdios cardiológicos no invasivos. In: Storino R, Milei J, editores. Enfermedad de Chagas. $1^{\text {nd }}$ ed. Buenos Aires: Division Mosby; 1994. p.359-454.

23. Brant TC, Laranja FS, Bustamante FM, Melo AL. Dados sorológicos e eletrocardiográficos obtidos em populações não selecionadas de áreas endêmicas de doença de Chagas no Estado do Rio Grande do Sul. Rev Bras Malariol D Trop 1957; 9:141-148.

24. Organización Mundial de la Salud. Enfermedad de Chagas. Informe de un grupo de estudio. Informe Técnico $n^{\circ} 202$. Ginebra: World Health Organization; 1960.

25. Organización Panamericana de la Salud. Enfermedad de Chagas. Informe de un grupo de estudio. Publicación Científica $\mathrm{n}^{\circ} 195$. Washington DC: Organização Panamericana de Saúde; 1970.

26. World Health Organization. Clinical aspects of Chagas disease. Report of a Pan American Health Organization and World Health Organzation meeting of investigators. Caracas: Pan American Health Organization; 1971.

27. Puigbó JJ, Nava Rhode JR, Barrios HG, Yepez CG. Cuatro años de estudio longitudinal de una comunidad rural con endemia chagásica. Bol of Sanit Panam 1969; 48:112-120.

28. Cichero J, Madoery R. Aspectos epidemiológicos de La enfermedad de Chagas-Mazza em La República Argentina. In: Posse RA, MouzoG, Barrio N editores. Enferemedad de Chagas. Buenos Aires: Ministério de Salud; 1981.

29. Rose A, Blackburn H. Cardiovascular survey methods. Geneva: World Health Organ. Monogr. Series 56; 1964.

30. Conselho Nacional de Pesquisas. Epidemiologia da doença de Chagas. Objetivos e metodologia dos estudos longitudinais. Brasília: Relatório Técnico $\mathrm{n}^{\circ} 1 ; 1974$.

31. Prata AR. Reunião sobre diferenças geográficas na doença de Chagas. Brasília: Universidade de Brasília; 1975.
32. Guimarães AC. Aspectos Eletrocardiográficos da Cardiopatia Chagásica Crônica. In: Cançado JR, Chuster M, editores. Cardiopatia Chagásica. Belo Horizonte: Fundação Carlos Chagas; 1984. p.141-147.

33. Programa de Salud Humana. Nomeclatura y Critérios de Diagnóstico Electrocardiográfico. Buenos Aires: Universidad Del Salvador; 1985.

34. Lázzari JO, Pereira M, Antunes CMF, Guimarães A, Moncayo A, Domínguez RCH. Diagnostic electrocardiography in epidemiological studies of Chagas' disease: Multicenter evaluation of a standardized method. Rev Panam Salud Publica 1998; 4:317-330.

35. Gonçalves JGF, Prata A. A comparison of three electrocardiogram coding systems for chronic Chagas' disease. Rev Panam Salud Publica 2003; 14:201-208.

36. The Criteria Committe of the New York Heart Association. Nomenclature and Criteria for Diagnosis of Diseases of the Heart and Great Vessels. Ninth Edition, Boston: New York Heart Association; 1994.

37. Maguire JH, Mott KE, Souza JAA, Almeida EC, Ramos NB, Guimarães AC. Electrocardiographic Classification And Abbreviated Lead System for Population-Based Studies of Chaga's Disease. Bull Pan Am Health Organ 1982; 16:47-58.

38. Coura JR. Reunião sobre diferenças geográficas na doença de Chagas. Brasília:1975; p.97-99.

39. Maguire JH, Mott KE, Hoff R, Guimarães A, Franca JT, Souza JAA, et al Three-Year Follow-Up Study Of Infection With Trypanosoma cruzi And Electrocardiographic Abnormalities in a Rural Community in Northeast Brazil. Am J Trop Med Hyg 1982; 31:42-47.

40. Pereira JB, Willcox HP, Coura JR. Morbidade da doença de Chagas III. Estudo longitudinal, de seis anos, em Virgem da Lapa, MG, Brasil. Mem Inst Oswaldo Cruz 1985; 80:63-71.

41. Maguire JH, Hoff R, Sherlock I, Guimarães AC, Sleigh AC, Ramos NB et al. Cardiac morbidity and mortality due to Chagas, disease: prospective electrocardiographic study of a Brazilian community. Circulation 1987; 75:1140-1145.

42. Mota EA, Guimarães AC, Santana OO, Sherlock I, Hoff R, Weller TH. A nine year prospective study of Chagas' disease in a defined rural population in Northeast Brazil. Am J Trop Med Hyg 1990; 42:429-440.

43. Baruffa G, Aquino Neto JO, Alcântara Filho A, Bettini VN, Bertinetti ES. Dados preliminares de inquérito sorológico e eletrocardiográfico para doença de Chagas em populações rurais não selecionadas da zona sul do Rio Grande do Sul. Rev Soc Bras Med Trop 1972; 6:362-363.

44. Porto CC. 0 eletrocardiograma no prognóstico e evolução da doença de Chagas. Arq Bras Cardiol 1964; 17:313-346.

45. Carrillo RP. Estudo longitudinal de 25 anos da doença de Chagas em Mambaí/Buritinópolis - (GO), Brasil [Tese]. [Brasília]: Universidade de Brasília; 2001.

46. Gonçalves JGF. Evolução clínica,eletrocardiográfica e estudo prognóstico de moradores de área endêmica, sob controle, de doença de Chagas [Tese]. [Uberaba]: Universidade Federal do Triângulo Mineiro; 2007. 